\title{
Autoimmune Nervous System Disorder
}

National Cancer Institute

\section{Source}

National Cancer Institute. Autoimmune Nervous System Disorder. NCI Thesaurus. Code C99383.

A disorder characterized by the degeneration of the nervous system due to autoimmunity. Representative examples include multiple sclerosis, Guillain-Barre syndrome, and myasthenia gravis. 